La terre lorraine, les puissants et les hommes : La plus ancienne charte française du scriptorium épiscopal de Toul (1237)

Glessgen, M D

Posted at the Zurich Open Repository and Archive, University of Zurich

ZORA URL: https://doi.org/10.5167/uzh-60854

Journal Article

Published Version

Originally published at:

Glessgen, M D (2010). La terre lorraine, les puissants et les hommes : La plus ancienne charte française du scriptorium épiscopal de Toul (1237). Bibliothèque de l’École des Chartes, 168:25-46. 


\title{
LA TERRE LORRAINE, LES PUISSANTS ET LES HOMMES : \\ LA PLUS ANCIENNE CHARTE FRANÇAISE DU SCRIPTORIUM ÉPISCOPAL DE TOUL (1237)
}

\author{
par \\ Martin-D. GLESSGEN
}

\section{AnaLYSES MaCroscopiQue ET MiCroscopiQue DANS LES PLUS ANCIENS DOCUMENTS.}

L'étude des chartes réunies dans la collection des Plus anciens documents linguistiques de la France, édition électronique, soulève l'opposition épistémologique entre les deux formes d'analyse linguistique possibles pour les textes anciens, macroscopique et microscopique. La première a pour vocation de prendre connaissance, dans la mesure du possible, de l'ensemble des sources disponibles pour une époque et une langue données et d'en retracer l'histoire interne et externe. L'analyse microscopique, en revanche, suppose le traitement minutieux de tous les éléments pertinents dans un texte ou manuscrit individuel, placé au mieux dans son contexte de genèse. Les deux mouvements se complètent puisque l'élaboration globale repose sur la somme des travaux dédiés à des textes individuels, qui à leur tour s'inspirent des interprétations globalisantes disponibles. Ils se trouvent néanmoins en opposition réelle dans la recherche concrète : il est tout aussi impossible de considérer en détail tous les textes si l'on doit en gérer plusieurs milliers à la fois que d'identifier tous les paramètres pertinents pour l'écrit d'une époque donnée lors de l'édition ou de l'étude par le menu d'un texte défini.

Les travaux philologiques se déploient donc toujours entre ces deux pôles, en même temps opposés et inséparables, en privilégiant l'un ou l'autre selon les interrogations poursuivies : c'est, en dernière instance, l'opposition qui règne entre d'une part la linguistique diachronique traitant

Martin-D. Glessgen, professeur de philologie romane et française à l'université de Zurich, Romanisches Seminar, Zürichbergstrasse 8, CH-8032 Zürich. <glessgen@rom. uzh.ch> 
la langue en tant que système évolutif (ou «configuration ») et d'autre part l'étude des textes définis en tant que discours ${ }^{1}$.

L'élaboration de notre base de données est, elle aussi, tributaire de cette opposition de fond : notre analyse porte à tour de rôle sur certains aspects ciblés présents dans l'ensemble du corpus (les auteurs ou bénéficiaires, les caractéristiques scriptologiques, des parcelles définies du lexique ou des éléments de syntaxe), et sur l'ensemble de ces aspects présents dans une charte définie. Les résultats obtenus dans chacune de ces opérations restent en même temps inévitablement partiels et sont soumis à des précisions et corrections ultérieures en fonction de l'avancement de la recherche.

La présente étude s'inscrit dans la logique d'une analyse microscopique. Sa première rédaction remonte à la phase préparatoire de nos travaux sur les chartes; elle visait à mieux cibler les interrogations macroscopiques qui seraient à mettre en œuvre par la suite. Nous avons donc mené, à partir d'une charte individuelle, enrichie d'un vidimus du même acte, une étude de faisabilité, en analysant les différents aspects qui semblaient significatifs, aux plans graphématique et lexical, diplomatique et historique.

Le choix du document, une charte épiscopale de Toul de 1237, se justifie par son ancienneté et par l'identification supposée sûre de son rédacteur. Par ailleurs, à tort ou à raison, le corpus des chartes épiscopales de Toul nous a semblé d'un intérêt particulier au début de notre recherche, et il a fait aussi l'objet du mémoire pour le diplôme d'études approfondies de Dumitru Kihaï ${ }^{2}$. Nous verrons par la suite que, pour la période antérieure à 1256, l'évêque ne s'exhibe que très exceptionnellement en tant qu'acteur dans l'arène des chartes vernaculaires, mais ce n'était pas prévisible avant d'avoir achevé l'identification des rédacteurs dans le corpus de Meurthe-etMoselle ${ }^{3}$.

Notre étude a toutefois été indispensable pour préparer cette identification, notamment pour déterminer les paramètres pertinents dans celle-ci et pour évaluer l'interaction entre les données linguistiques et extralinguistiques. L'étude macroscopique des rédacteurs a permis ensuite de mieux cerner la nature des caractéristiques graphématiques de notre charte et de mieux fonder sa comparaison diplomatique avec les autres documents contemporains témoignant d'une intervention de l'évêque.

1. Voir M.-D. Gleßgen, «Philologie und Sprachgeschichtsschreibung in der Romanistik : die "informatische Wende" », dans Edition und Sprachgeschichte: Baseler Fachtagung, 2.4. März 2005, éd. Michael Stolz, Tübingen, 2007 (Beihefte zur Editio, 26), p. 201212, et ci-dessus, « Présentation générale », note 40.

2. Dumitru Kihaï, Identification des rédacteurs d'un corpus de chartes lorraines du XIII ${ }^{e}$ siècle, mémoire présenté à l'Université Marc-Bloch de Strasbourg, 2003.

3. Voir M.-D. Gleßgen, « Les "lieux d'écriture" dans les chartes lorraines du xiI" siècle », dans Revue de linguistique romane, t. 72, 2008, p. 413-540. 
Il est donc heureux que la publication, prévue initialement en 2004, ait été retardée d'année en année, puisque nous découvrions continuellement de nouvelles facettes qui nuançaient ou accentuaient les premiers constats ${ }^{4}$. Après l'achèvement du premier volet de notre projet ${ }^{5}$, les doutes encore persistants dans l'étude de la charte de 1237 ont presque tous pu être levés et l'analyse microscopique repose désormais sur un nombre important de données. On trouvera donc ci-après l'édition, suivie de ses lectures lexicologique, graphématique, diplomatique et, enfin, historique.

\section{Description ANALYTiQue et ÉDition De LA ChaRte.}

La charte fortement élaborée de 1237 contient d'emblée des indices forts qui plaident pour une rédaction par le scriptorium épiscopal de Toul :

(1) Tous les protagonistes de la charte (auteur, bénéficiaire de l'acte, transmission) indiquent ce scriptorium : l'évêque est l'auteur et le bénéficiaire de la charte, qui est conservée aujourd'hui dans le fonds de l'évêché aux archives départementales de Meurthe-et-Moselle.

(2) Plus précisément, il s'agit d'une charte d'arbitrage ${ }^{6}$ à la suite d'un différend qui opposa, par personnes interposées, deux des princes les plus puissants de la région, à savoir l'évêque de Toul et le duc de Bar. La charte et son vidimus, produit deux ans plus tard, sont destinés à renforcer la position de l'évêque (raison pour laquelle il faut considérer celui-ci comme bénéficiaire de l'acte).

(3) La mise en page est exceptionnellement maitrisée (le texte est placé dans un carré parfait avec de larges marges, le support est réglé) et l'écriture, quelque peu statique, est fortement ornée et travaillée : or en Lorraine, seuls les scriptoria épiscopaux produisent des documents semblables,

4. La publication était prévue dans un volume de mélanges pour le $60^{\mathrm{e}}$ anniversaire de Gilles Roques, volume qui pour des raisons qui m'échappent n'a pas encore vu le jour. C'est à l'occasion d'un déplacement avec Gilles Roques (qui devait nous mener en 1998 au congrès Urkundensprachen im Grenzbereich, à Trèves) que j'ai eu pour la première fois l'idée d'une approche informatique de l'ancienne langue et la puissance visionnaire de cet érudit ami m'avait alors fortement impressionné. Le choix du document, qui paraîtra ici à titre personnel en l'honneur de son $65^{\mathrm{e}}$ anniversaire, évoque en même temps le monde rural lorrain qu'il connaît si bien grâce à ses promenades cyclistes : ces terres lourdes et frustes des bords de la Meuse, ces collines et ces forêts sauvages, ces ruisseaux qui ont creusé en profondeur le terrain calcaire, tout évoque encore les habitants d'antan qui se disputaient violemment ces terres aujourd'hui si dédaignées.

5. Marqué par la mise en ligne des premières séries de chartes et ma première analyse systématique des lieux d'écriture.

6. La note dorsale de la charte indique : « karta de pace». 
ce qui permet d'exclure comme rédacteurs les grandes chancelleries laïques de Bar et de Lorraine, sans parler des lieux d'écriture mineurs ?

En l'absence d'autres protagonistes épiscopaux mentionnés, les critères externes et de contenu permettent donc un rattachement fiable au scriptorium épiscopal de Toul, ce qui offre en même temps un ancrage solide à l'analyse linguistique.

L'édition de la charte ci-après reproduit celle de la série des Chartes de la Meurthe-et-Moselle dans le corpus électronique des Plus anciens documents $^{8}$. À la différence de l'édition électronique, qui contient la transcription complète tant de la charte $n^{\circ} 7$ en question que du vidimus $\left(n^{\circ} 11\right)$, les variantes de ce dernier sont ici incluses dans l'apparat critique.

Notre édition électronique repose pour la Meurthe-et-Moselle sur une première édition imprimée de Michel Arnod établie d'après les critères de l'ancienne collection des Plus anciens documents (1974) ${ }^{9}$. Cette édition, bien que de très bonne qualité, a connu une réélaboration intense entre 2001 et 2008 afin de l'intégrer dans la logique de nos critères éditoriaux actuels, qui répondent au concept d'un encodage double (médiéval et moderne) ${ }^{10}$. Les interventions concernent principalement :

(1) la prise en considération des éléments originaux de marquage paléographique (ponctuation, majuscules, séparation des mots);

(2) l'intégration d'une structuration explicite par des numéros en gras;

(3) un élargissement du tableau analytique incluant notamment le rédacteur (potentiel ou assuré) de la charte;

(4) un nombre non négligeable de corrections de détail dans la transcription (en moyenne, deux corrections par charte) ${ }^{11}$;

(5) une attention particulière à la gestion des noms propres et des titres (majuscules, ponctuation);

(6) une reproduction en photographie numérique, recto et verso.

Des développements ultérieurs nous sembleraient utiles dans toutes les séries actuellement mises en ligne, en ce qui concerne le regeste. Celui-ci

7. L'argumentation présente repose sur les résultats méthodologiques et concrets des « lieux d'écriture » (voir note 3).

8. Les plus anciens documents linguistiques de la France, édition électronique : les chartes de la Meurthe-et-Moselle (ChMM), dir. M.-D. Gleßgen, transcription et édition électronique par M.-D. Gleßgen et Michel Arnod, relecture par Dumitru Kihaï (1232-1265 : 290 doc.). Voir aussi la reproduction photographique de la charte dans M.-D. Gleßgen, « Les "lieux d'écriture"... », p. 530.

9. Michel Arnod, Publication des plus anciennes chartes en langue vulgaire antérieures à 1265 conservées dans le département de Meurthe-et-Moselle, thèse de $3^{\mathrm{e}}$ cycle, Université Nancy-II, 1974, dactyl.

10. Voir ci-dessus, « Présentation générale... », III, 2, 1, no 1 .

11. L'édition qui suit indique les leçons de l'édition de M. Arnod. 
devrait être élargi pour mieux expliciter les contenus des différentes chartes, souvent très denses, implicites et entremêlés.

Le cas de la Meurthe-et-Moselle montre bien que la transcription en tant que telle ne représente qu'un premier pas dans l'élaboration éditoriale des documents, sans parler des questions d'analyse linguistique et d'identification toponymique ${ }^{12}$.

Par ailleurs, la charte $\mathrm{n}^{\circ} 7$ avait déjà connu une édition antérieure dans la thèse de M. Scott (1924) ${ }^{13}$. Elle a aussi été reproduite sous forme de facsimilé, accompagnée d'un commentaire principalement paléographique, dans le catalogue de l'exposition La plume et le parchemin ${ }^{14}$. Le document est donc connu et a pu être utilisé par la recherche historique et linguistique. Notre édition ajoute toutefois des éléments nouveaux qui en permettent une exploitation plus approfondie.

Elle intègre, comme nous l'avons dit, les variantes du vidimus. Celui-ci, émis sous l'autorité du doyen du chapitre, Pierre, reproduit très exactement la charte initiale; il y ajoute un préambule (1-5) qui rappelle l'opération de l'arbitrage ainsi qu'une nouvelle corroboration (22 sq.), qui sont édités séparément à la suite du document.

La structure sémantique de la charte est la suivante :

(1) Présentation de l'auteur, Roger, évêque de Toul, (3) et des quatre arbitres (4) choisis par les deux parties, le chapitre de Toul et Gaucher de Commercy; (5) évocation du différend à régler, les torts faits par Gaucher au chapitre par suite de la prise en garde comtale d'une terre du chapitre, (6) et du rôle de l'évêque, élu rapporteur et arbitre suprême ; (7) description du terrain en cause (10) et de son emplacement; (12) les règles de l'accord : le seigneur de Commercy doit rendre les serfs qu'il a pris à son propre service, (13) mais des réclamations restent possibles (14) tant que le chapitre n'aura pas restitué à son tour les redevances qu'il a perçues à tort. (1517) Corroboration.

12. Il importe de souligner que l'édition de M. Arnod et les photocopies des documents qu'il m'a aimablement transmises en 2001 ont été d'une inestimable utilité au début de mes travaux. Ils ont permis notamment de former une équipe de jeunes chercheurs aux techniques de transcription, d'identification de toponymes et de description des documents; la collection de Meurthe-et-Moselle contient des cas de figure très divers et des chartes souvent difficiles à transcrire et à comprendre. En réélaborant les données déjà très travaillées et fiables de $\mathrm{M}$. Arnod, mes élèves ont pu acquérir la sécurité de jugement nécessaire pour entreprendre la rédaction autonome d'autres séries et la réélaboration d'autres corpus anciens.

13. M. Scott, Chartes du XIII ${ }^{e}$ s. en dialecte toulois, thèse de doctorat, Université de Nancy, 1924, dactyl., $\mathrm{n}^{\circ}$ IV.

14. Simone Collin-Roset et al., Écriture et enluminure en Lorraine au Moyen Âge, catalogue de l'exposition «L L plume et le parchemin » organisée par la Société Thierry-Alix, Nancy, 1983, p. 36. 


$$
007-1237 \text {, juin }
$$

AD MM 2 F 2, $n^{\circ}$ 14, évêque de Toul.

Parchemin jadis scellé sur double queue, sur lacs de soie. $178 \times 313 \mathrm{~mm}$.

1 Nos, Rogiers, par la grace Deu aveques de-Toul ${ }^{\text {a }}, 2$ fasons conoxant a-toiz ceaus qui ses leitres ${ }^{\mathrm{b}}$ voiront $3 \mathrm{ke} \mathrm{li}$ arcediacres / Girars et Symons de Ramonchamp, chanenne $^{\mathrm{c}}$ de Toul, et li sires Bertremeus de Apremont et Phelipes de Euvile, chivalier, / 4 qui furent eilui ${ }^{1}$ disor de-par le chapitre de Toul et le seignor Gaucher de Commarcei, 5 de-toz le tors ke li chapitre ${ }^{2}$ disoit qu'il / lor avoit fait depeus ke li cuens Henris de Bair qui est ${ }^{3}$ prist lor terre en garde, 6 raporterent par concort en nostre presence, ki estiens // ellui apardesuis c'il ce decordoient, 7 ke toites le terres et li boisson, et les espines, et prei, et pastures 8 ke sont entre le ruxel devers Chair/nai qui ai nom »Ruus « et la premiere ${ }^{\mathrm{d}}$ bonne k'il mirent en l'oriere do Haut Bois, 9 tot contremont juske a-champ ke cil de Reevaus / tienent do chapitre de Toul et, contreval, juke a-la-voie ke vient de Commarcei a-Voi, 10 et par desus le bonnes k'il ont assises par davant / Reeval juqe de soz la Horne, ver Voi et devers le dependant, apartienent a-chapitre de Toul; 11 et il le revestirent de quanque li sires / de Commarcei lor avoit fait tort dedens le bonnes; 12 et ce $^{4}$ lo revestirent de deus homes de kes li uns ai nom Girboles et li autres // Undeneis, ki sont a-Voi menant, 13 en tel meniere ke ce ${ }^{5}$ li chapitres et li sires de Commarcei se welent de ces-tenures et d'autres riens de/mander, il le poiront faire ou il deverent, 14 fors tant ke li chapitres at aquitei le seignor ${ }^{f}$ de Commarcei de toiz les chateiz k'il disoit k'il / avoit pris a-tort en lor homes de-Voi; $15 E t$ nos, le raport et les choises ${ }^{g}$ ke sont davant escrites, crantonz et confermons. $16 \mathrm{Et}$ por ce ke ce / soit estaible ${ }^{\mathrm{h}}$ choise, avons mis nostre seel en teimongnaige de vertei .

17 Ce fuit fait en l'an ke li miliares corroit par mil et / deuz ${ }^{i}$ cens et trente seit ${ }^{j}$ ans, en mois de junet. ${ }^{k}$

${ }^{\text {a }}$ Vid. de Toul, de même que de par 4 , de toz 5 , de peus 5 , de vers $8 ; 10$, de pendant 10 , de Voi 14. ${ }^{\mathbf{b}}$ Vid. letres. ${ }^{\mathbf{c}}$ Vid. chanoinne. ${ }^{\mathbf{d}}$ Avec un signe d'abréviation redondant pour -re-. ${ }^{\mathbf{e}}$ Vid. juque. ${ }^{\mathbf{f}}$ Vid. signor. ${ }^{\mathbf{g}}$ Vid. choses. ${ }^{\mathbf{h}}$ Vid. estable. ${ }^{\mathbf{i}}$ Vid. douz. ${ }^{\mathbf{j}}$ Vid. set. ${ }^{\mathbf{k}}$ en mois de ju net : étalement des mots sur la fin de la ligne.

${ }^{1}=e ́ l u .{ }^{2}$ Pour li chapitres (c.suj.). ${ }^{3}=$ qui est actuellement en titre. ${ }^{4}=s i .{ }^{5}=s i$.

Vidimus : 011 - 1239, avril

AD MM 2 F 2, $n^{\circ} 15$, évêque de Toul.

Parchemin jadis scellé sur double queue.

1 Nos, Pierres, li doens et tus li chapitres de Toul, 2 fasons conoxant a-toiz ceaus qui ses letres voiront 3 ke nos le raport que li arcedia/cres Gerars et Symons de Ramonchamp, nostre chanoinne, et li sires Bertremeus de Apremont et Phelipes de Euvile, chivalier, 4 qui furent eilui / disor de nus d'une part et de signor Gaucher de Commarcei d'autre, 5 de toz le tors que nus disiens qu'il nus avoit fait des peus ke li cuens Hen/ris de Bar qui est prist nostre terre en garde, ont raportei par concort, creantons et loons en tel maniere com li rapors est escris en letres mon 5 signor // Rogier, avesque de Toul, qui sunt itels . 
6 Nos, Rogiers (...) [= suit le texte de la charte originale]

22 Et por ce que ce soit plus ferme chose, nos, li doens et li chapitres de Toul, avons donees audit Gaucher letres saelees de nostre / seel · 23 en l'an ke li miliares corroit par mil et douz cens et trente nieuf, en mois de avri .

\section{LE LEXIQUE D'APRÈS SES CHAMPS SÉMANTIQUUES.}

L'étape la plus délicate pour étudier un document en profondeur est la compréhension de son contenu textuel, qui suppose de définir chaque mot individuel. Une telle opération reste pour la plupart implicite, surtout dans un cas comme celui-ci, puisque le lexique de la charte ne réserve aucune surprise : il s'agit de termes de l'ancienne langue, habituels dans le genre textuel en question et bien connus grâce au Dictionnaire de Frédéric Godefroy ${ }^{15}$. Mais le travail lexicologique ne saurait se passer d'une étape préalable, l'analyse exhaustive d'un certain nombre de chartes, avant d'aborder l'étude de questions plus spécifiques.

Par souci de transparence, figure donc ci-dessous une présentation glossographique des mots qui portent la charge sémantique du texte, moins pour élargir notre connaissance de l'ancienne langue que pour garantir une bonne compréhension de la charte en question ${ }^{16}$. Les mots grammaticaux et les lexèmes de base (faire, estre, avoir, voir, tenir, homes, nom) ont été laissés de côté, de même que les termes et collocations très courants du langage juridique ${ }^{17}$.

Les autres termes ont été organisés selon leurs champs sémantiques : le monde agricole (boisson, espines, etc.), y inclus celui de sa gestion et de son droit (bonnes, dependant), les domaines confondus de la diplomatique (letres saelees), du droit (disor) et de la société féodale (chivalier), ainsi que la hiérarchie ecclésiastique (arcediacres, avesques, etc.); ce dernier aspect appellera quelques commentaires historiques ultérieurs puisqu'il importe dans les choix concernant les lieux d'écriture ecclésiastiques de Toul à cette époque.

15. Voir Frédéric Godefroy: actes du $X^{e}$ colloque international sur le moyen français (Metz, 12-14 juin 2002), éd. Frédéric Duval, Paris, 2003.

16. Les renvois se limitent ici essentiellement aux dictionnaires de Godefroy et du FEW, cités dans un ordre variable, selon la pertinence des informations qu'ils apportent ; la bibliographie spécifique sera intégrée dans la base lexicologique.

17. Cf. (d'autres riens) demander «réclamer, demander 》 v.tr. 13, estaible choise «immuable, établi »16, fasons conoxant «faire connaître, rendre connu » 2, ferme chose «immuable, fixe» vid. 22, leitres n.m.pl. «charte» 2, letres vid. 2, 5, 6, 22, loons v.tr.prés.6 « approuver 》 vid. 5, menant p.prés. «logé, résidant » 12 , quanque adv. « tout ce que » 10 , saelees adj.f.pl.rég. « scellé » vid. 22 , seel n.m.rég. «sceau » 16 , en teimongnaige n.m.rég. 16. 


\section{Le monde agricole, sa gestion et son droit :}

assises (bonnes ) v.tr.p.pass. « placer, poser (un objet) $\gg 10$ (= vid.) ${ }^{18}$; le terme ne possède donc aucune spécificité sémantique, même s'il est introduit dans un contexte de gestion rurale; cf. FEW 12,398b s.v. SEDĒRE : asseoir BenSMh-Oud $1660 ;$ Gdf 8/2,200 sq.

boisson n.m.pl.suj. «touffe d'arbrisseaux ou d'arbustes sauvages » 7 (= vid.); cf. FEW 15,196a s.v. *bosk: boisson (Roland-RoseM), buisson (GlOxf; Wace; dp. 1190ca.) etc.; Gdf 8/2,353a-c (« exprime dans les anciens textes bien plus l'idée de petit bois... que celle de buisson »).

bonne n.f.rég. «borne miliaire» 8, bonnes pl.rég. 10 sq. (= vid.); cf. FEW 1,465 a s.v. *botina; mot avec une forte variation grapho-phonétique (bo(u)ne, bosne, bodne, bonde, etc.; cf. pour bonnes 1274, ms. fin $13^{\mathrm{e}}$ s., ChronSDen, Gdf $8 / 2,334 \mathrm{~b})$.

champ n.m.rég. « portion de terre labourable » 8 (= vid.).

chateiz n.m.pl.rég. «jouissance ou perception des fruits d'un travail [Gdf]» 14 (= vid.) ; cf. FEW 2,253b s.v. CAPITALIs : chatel « biens mobiliers, patrimoine » $\left(12^{\mathrm{e}}-15^{\mathrm{e}}\right.$ s.) etc.; Gdf $2,89 \mathrm{~b}$ propose comme sens alternatif « jouissance ou perception des fruits d'un travail » (éventuellement sous forme d'argent); dans les nombreuses attestations (ibid., 89b-90b), les deux sens alternent, sans qu'une distribution diasystématique soit clairement reconnaissable; dans notre contexte, il s'agit du deuxième sens, puisque le seigneur de Commercy demande au chapitre de l'investir (aquitei) des chateiz que le chapitre aurait pris a-tort en lor homes de-Voi: les hommes appartiennent au chapitre, mais ils doivent au seigneur de Commercy des redevances perçues « à tort » par le chapitre ; s'il avait été question de saisies de biens, ceux-ci auraient dû être rendus au paysans.

contremont (tot $\sim)$ adv. «en amont » 9 (= vid.); cf. Gdf 9,184b; FEW 2,112b (Roland-Ac 1878).

contreval adv. « en aval » 9 (= vid.); cf. Gdf 2,271a s.v. contraval; 281b; FEW (Roland-Oud 1660).

dependant n.m.rég. «terres qui relèvent d'un lieu habité » 10 (= vid.); terme mal répertorié dans Gdf $(2,513 \mathrm{a} / \mathrm{b} ; 9,305 \mathrm{c} ; 351 \mathrm{~b})$ et par conséquent dans le FEW (8,181b/182a s.v. PENDĒRE); c'est un adj. substantivé (le sens adj. le plus proche, « qui relève d'un autre, en parlant d'un fief », est daté 1510-Ac 1798, ibid. 182a) provenant du verbe dependre v.intr. «relever de (en parlant d'un fief)» (bourg. 1459, Perche 1505, ibid. 181b).

espines n.f.pl.rég. « arbre ou arbrisseau dont les branches ont des piquants » 7 (= vid.) ; cf. FEW 12,177b s.v. sPĪNA : Passion-Trév 1771; Gdf 9,545a.

oriere n.f.rég. « bordure, lisière (d'un bois) » 8 .

pastures n.f.pl.rég. «lieu où l'on fait brouter le bétail, pâturage » 7 (= vid.); cf. FEW 7,763a s.v. PASTŪRA : $12^{\mathrm{e}}$ s.-1619; Gdf 6,36c; 10,294a.

prei n.f.pl.rég. «petite étendue de terre où l'on fauche l'herbe » 7 (= vid.); cf. Gdf 10,299c; FEW 9,333b.

18. L'indication «= vid.» dénote que la même forme apparaît au lieu correspondant du vidimus; si la forme intervient en d'autres lieux du vidimus, cela est indiqué par «+ [chiffre] $»$, renvoyant au paragraphe du vidimus. 
ruxel n.m.rég. « cours d'eau moins large qu'une rivière » 8 (= vid.); cf. FEW 10,424b s.v. RīvUSCELlus : ruissel (Wace-1382)... ruxel (lothr., RF 5,597) etc.; Gdf $10,610 \mathrm{a} / \mathrm{b}$.

tenures n.f.pl.rég. «bien, propriété, domaine, terres qu'on tient comme fief »13 (= vid.) ; cf. FEW 13,220a s.v. TENĒRE : teneure (12 s.), tenure 1274-Oud 1660 ; la date de 1274 [= ALoiret, Gdf 7,680b] peut être remplacée par celle de notre charte; par ailleurs, dans notre contexte $t$. se réfère autant à des hommes qu'à des terres et redevances.

terres n.m.pl.rég. « sol considéré par rapport à la culture » 8 (= vid.) ; cf. FEW 13,245a : dp. 1283 [ms. ca. 1300], BeaumCoutS, tarre (1252, RegTempl, Gdf $10,757 \mathrm{c})$; notre charte fournit une attestation légèrement plus ancienne de ce sens (néanmoins difficile à séparer de « sol sur lequel nous marchons... qui nourrit les végétaux » [Passion; dp. Alexis, ibid.] et des autres sens métonymiquement très proches); le sens de «champ (opp. à pré) » est tardif (1636-1715, FEW ibid./24.5b).

\section{Diplomatique, droit et féodalité :}

aquitei[ $r]$ (qn de qch), aquitei v.tr.p.pass. « payer sa dette (à qn) » 14; la structuration sémantique dans FEW 2,1472a/1473a s.v. QUIĒTus est à revoir; dans cette utilisation, précise seulement : aflandr. 1280 ; cf. Gdf 8/2,162b/c.

concort (par $\sim)$ 《 (en) accord 》 6 (= vid. + 5); cf. FEW 2,1012b s.v. conconDARE : concort «accord (t. de droit)» $\left(13^{\mathrm{e}}-16^{\mathrm{e}} \mathrm{s}\right.$.) ; cf. Gdf 2,222c par comun concort (1260, AMeurthe).

crant [eir], crantons v.tr.prés. 6 « approuver, permettre, consentir » 15 (= vid.), creantons vid. 5.

[se] decord[eir], (ce) decordoient v.pron.impf.6 «entrer en désaccord》 6 (= vid.); cf. Gdf $2,566 \mathrm{~b} / \mathrm{c}$.

disor n.m.pl.rég. « arbitre » 4 (= vid. + 4) : cf. disoirs (1233, ArchLiège), diseur (1235, ArchNord), disor (1242, ArchMeurthe ${ }^{19}$ ) etc. [-15 ${ }^{\mathrm{e}}$ s.], Gdf 2,722a/b; [FEW 3,68a s.v. DĪCERE seulement le sens fr. diseur « celui qui dit habituellement certaines choses $\gg]$.

garde (prist en $\sim$ ) « surveillance, protection militaire d'un territoire ou d'un lieu habité par un seigneur féodal, moyennant une redevance » $5(=$ vid. +9$)$; suite à Gdf $(9,684 \mathrm{a} ; 4,223 \mathrm{a}), \mathrm{FEW}(17,515 \mathrm{~b}$ s.v. * wardôn) répertorie le sens général « surveillance, protection, soin qu'on prend de qch » (dp. Alexis [guarde]; Wace) [= DEAF G 158] ainsi que de nombreux sens spécialisés et syntagmes; le sens précis de notre charte et la collocation (prendre en garde) n'apparaissent pas; DEAF G 160 préconise le sens «droit de protection sur un lieu (exercé par un seigneur ou par la justice) », mais ne donne qu'une seule attestation (hbret. ca. 1320); l'usage dans notre charte doit être rapproché des attestations plus nombreuses notamment lorraines que le DEAF réunit (ibid.) sous le sens « droit de protection sur une abbaye, une église »; mais il s'agit bien de la protection d'un bien, non pas d'une église ${ }^{20}$.

19. Gdf reproduit ici fidèlement l'essentiel de la charte 18 (3-7) de mon corpus, avec de légères modernisations : devisé pour devisei, deux pour deuz, et l'introduction erronée d'un $i$ parasite : boine pour bone).

20. C'est aussi le sens de la prise en garde des territoires de l'universitas de Toul par le comte de Bar, survenue elle aussi au début de l'année 1237 (Gerold Bönnen, Die Bischofs- 
junet n.m.rég. « juin » 17 (= vid.), régionalisme ${ }^{21}$.

miliares n.m.suj. «millénaire » 17 (= vid. +23), régionalisme ${ }^{22}$.

rapors n.m.suj. « récit, rapport qu'on fait » vid. 5, raport rég. 15 (= vid. +3); déverbal du suivant; cf. FEW 9,209a s.v. PORTARE : report (ca. 1200, SJeanBoucheD ; 1530, Palsgrave), et Gdf 10,482b : raipors (1214, CollLorr), rapoirs (1291, AMeuse) etc.

raporte[ir], raporterent v.tr.prét. 6 «faire le récit d'un fait, faire un rapport sur une affaire » 6 (= vid.), raportei p.perf. vid. 5 (raport que... ont $\sim$ ); sens mal répertorié dans le FEW 9,208b [seulement pour bearn.], par suite de son absence en $\operatorname{Gdf}(10,482 \mathrm{c}-483 \mathrm{~b})$.

revestir, revestirent (qn de qch) v.tr. «mettre en possession »11, 16 (= vid.); cf. FEW 14,354a : revestir (qn de qch) (Chrestien-Trév 1771).

tort n.m.rég. « acte qu'on ne devrait pas faire » 10 (= vid.), tors pl.rég. 5 (vid. torz $+5)$.

a-tort adv. 13 (= vid.) ; syntagme non relevé dans FEW 13,87a/b s.v. TORQQUĒRE ; mais cf. Gdf 10,780b : a tort (13 s., BibleGuiot).

\section{La hiérarchie ecclésiastique :}

arcediacres n.m.pl.suj. «premier dignitaire de l'évêché après l'évêque, archidiacre»23 3 (= vid.); type habituel de l'a.fr., cf. archediacre (1174ca., SThomGuern, FEW 24,95a s.v. ARCHIDIACon [mais Gdf indique pour ce texte arcediaknes, arcediakene $]-16^{\mathrm{e}} \mathrm{s}$ ), arcediacres (1248, Thouars, AVienne, Gdf $8 / 2,169 \mathrm{c})$.

stadt Toul und ihr Umland während des hohen und späten Mittelalters, Trèves, 1995, p. 406) ; les liens entre ville et évêché dans cette opération parallèle ne sont pas clairs.

21. Voir Jacques Monfrin, « Notes lexicographiques, II : Aprés la feste sain Johan, en juignet (Villehardouin 490) », dans Mélanges de linguistique française et de philologie et littérature médiévale offerts à M. Paul Imbs, Strasbourg, 1973, p. 157-168.

22. Voir M.-D. Gleßgen, «Das altfranzösische Geschäftsschrifttum in Oberlothringen : Quellenlage und Deutungsansätze », dans Skripta, Schreiblandschaften und Standardisierungstendenzen: Urkundensprachen im Grenzbereich von Germania und Romania im 13. und 14. Jahrhundert, Beiträge zum Kolloquium (16.-18. September 1998), éd. Kurt Gärtner, Günter Holtus, Andrea Rapp et H. Völker, Trèves, 2001, p. 264-265, à la p. 281.

23. L'archidiacre n'est pas, à l'origine, prêtre mais diacre (n'ayant pas reçu les ordres majeurs), ce qui permettait de minimiser d'éventuels conflits de pouvoir avec l'évêque. Il est responsable de la supervision du clergé, notamment des doyens de chrétienté (doiens de la crestientei [passim dans le corpus de $\mathrm{MM}$ ]; lat. decani rurales), à leur tour préposés aux différents prêtres d'une paroisse ou d'un ensemble de paroisses formant un chapitre rural (en territoire d'Empire, le doyen de chrétienté est élu par son chapitre rural). L'archidiacre peut aussi intervenir dans la supervision de la juridiction ecclésiastique ; à Toul, l'official (officials de la cort de Toul : passim dans le corpus de MM), chargé de l'exercice judiciaire du diocèse, répond non pas directement à l'évêque, mais à un archidiacre. À titre indicatif, en 1402, le diocèse de Toul comptait six archidiacres, vingt-trois doyens de chrétienté et six cent quatrevingts prêtres; le corpus mentionne à l'époque de Roger trois archidiacres : Gérard (dans le document ici étudié), Richier (ch. 17, 1242; ch. 31, 1243/1244) et Jacques (ch. 71, 1250), voir ci-dessous, IV, 2. Je remercie Benoît-Michel Tock des précisions fournies par lui sur les fonctions de l'archidiacre. 
aveques n.m.suj. « dignitaire élu ou nommé, chargé de la conduite d'un diocèse, évêque » ${ }^{24} 1$ (= vid.), avesque rég. vid. 5 ; variante habituelle de la prétonique ; cf. Gdf 8/2,575c/576a.

chanenne n.m.suj. « membre du chapitre d'une église cathédrale ou collégiale, chanoine (séculier) » 3, chanoinne vid. 3, 7 ; la variante tonique en - $e$ - n'est pas attestée à côté de chenoine, chanoine, etc. en Gdf 9,39a/b et FEW 2,218a.

chapitres n.m.suj. «corps des chanoines d'une église cathédrale ou collégiale » 2518 sq. (+ vid. 1, 22), chapitre 5 (= vid.), rég. 4, 9f; cf. Gdf 9,44b; FEW $2,265 \mathrm{~b}$ (dp. $12^{\mathrm{e}} \mathrm{s}$.).

doens n.m.suj. «dignitaire ecclésiastique préposé à un chapitre d'église cathédrale ou collégiale, doyen ${ }^{26}$ vid. 1, 22 (d. et tus li chapitres de Toul); cf. FEW (3,22b s.v. DECANUS : seulement la définition générale «dignitaire ecclésiastique ») et Gdf (9,293a, dans notre sens une seule attestation [1264, chap. Noyon, AOise]) ainsi que AND (1,142a s.v. dean : le d. et chapitre...).

4. Observations sur le lexique. - Le relevé lexical souligne surtout la dépendance étroite qui lie le FEW au dictionnaire de Godefroy ainsi que la grande richesse de celui-ci; comme J.-P. Chauveau l'a déjà montré ${ }^{27}$, le FEW place les mots dans leur histoire, mais il n'exploite pas toujours à fond les données du Godefroy ${ }^{28}$ et ne pallie pas ses lacunes ponctuelles pour le vocabulaire des sources documentaires ${ }^{29}$.

L'essentiel des mots de la charte appartient au langage général. Ils ont une faible spécificité sémantique et apparaissent dans des genres textuels très divers; cela vaut notamment pour presque tout le vocabulaire agricole et même ecclésiastique, mais aussi pour une bonne part du vocabulaire juridique (cranteir, leitres, tort, etc.). D'autres termes sont diaphasiquement marqués, plus spécifiques et - d'après les attestations anciennes propres à certains genres ou à certains contextes. Cela est surtout vrai pour le vocabulaire juridique, diplomatique et féodal mais aussi pour le vocabulaire de gestion agricole où quelques mots semblent être plus marqués que d'autres, comme chateiz, dependant, garde ou tenures. De même, dans le vocabulaire ecclésiastique, arcediacres et doens connaissent une diffusion

24. Le rôle de l'évêque est souvent politique, voire seigneurial, notamment dans une ville comme Toul où il détient les privilèges de battre monnaie, du marché et des taxes ; en 1261, le comté de Toul est transmis à l'évêque.

25. Le chapitre avait des charges liturgiques et pouvait conseiller l'évêque; à Toul, il accueillait jusqu'à soixante chanoines de la petite noblesse ou du patriciat régional, y compris les archidiacres; aux XII ${ }^{\mathrm{e}}$ et XIII $^{\mathrm{e}}$ siècles, le chapitre élisait l'évêque parmi la noblesse lorraine.

26. Le doyen était élu par les chanoines; parfois il se subordonnait à un prieur, mais ce n'est pas le cas ici.

27. Jean-Paul Chauveau, «L'utilisation du Dictionnaire de F. Godefroy dans le FEW », dans le colloque Frédéric Godefroy..., p. 323-344.

28. Par exemple pour les lexèmes chateiz, par concort, disor, raport, a tort; une nouvelle étude s'imposerait aussi pour aquite [ir] et arcediacres.

29. Par exemple pour les mots dependant, doens, garde, raporte[ir], tenures, terres. 
plus limitée dans les genres textuels que, par exemple, avesque ou chanoine. La distinction n'est pas toujours facile à opérer, mais elle a son importance pour juger de l'impact communicatif du texte.

\section{RÉgionalité LINGUISTIQUe : \\ LANGUE ET FONCTION DU TEXTE.}

La régionalité linguistique fournit un paramètre utile pour déterminer le degré d'élaboration des textes documentaires français au XIII ${ }^{\mathrm{e}}$ siècle. Fondamentalement suprarégionaux et latinisants, ces textes contiennent des marques diatopiques qui dénotent comment les rédacteurs se positionnaient dans l'espace sociogéographique de leur temps et quels modèles ils avaient choisi de suivre. Il s'agit bien entendu d'éléments régionaux à l'intérieur d'un type de langue élaboré et semi-artificiel, qui se distinguait en tout lieu clairement des dialectes parlés; les formes écrites pouvaient être transposées à l'oral dans des formes plus localisées, mais la distance interne entre les deux registres était nette.

1. Les marques graphématiques, morphologiques et lexicales. - Dans la charte d'arbitrage en question, les marques grapho-phonétiques régionales sont particulièrement présentes ${ }^{30}$. La plupart d'entre elles ont une valeur essentiellement ou purement graphématique ${ }^{31}$ :

$-<\mathrm{k}>$ au lieu de $<\mathrm{qu}>$ dans les fréquents pron.rel. : ke 3, 5, 7 etc. (12 fois, exclusif), $k i$ 5, 6 (contre 4 fois qui);

- <i> 'parasite', présent dans presque toutes les lignes de la charte : apardesuis « au dessus de » 6 (= vid.), Bair 5, choise(s) « chose(s) » 15 sq., eilui « élu » 4 (= vid. + 4), ellui 6 (= vid.), estaible 16, leitres 2, poiront «pourront» 13 (= vid.), seit « sept »17, teimongnaige 16 (= vid.), toiz 2, 13 (= vid. + 2), toites 7 (= vid.) [mais toz 5 (= vid. +5), tot 8 (= vid.)];

$-<\mathrm{w}->$ initial hypercorrect pour $v$-d'origine latine (welent 13 [= vid.]);

$-<\mathrm{x}>$ au lieu de $<$ ss $>$ : conoxant 2 [= vid. +2 ], ruxel 8 [= vid.] ${ }^{32}$.

Parmi les formes phonétiques régionales ressortent surtout deux évolutions dans le vocalisme tonique :

- la diphtongue /ej/notée <ei> comme résultat de la diphtongaison de A tonique en syllabe ouverte ainsi que de l'évolution du suffixe toponymique -iacu- : aquitei

30. Les chiffres se réfèrent aux divisions introduites dans l'édition. L'indication « = vid. » dénote que la même forme apparaît au lieu correspondant du vidimus ; si la forme intervient en d'autres lieux du vidimus, cela est indiqué par « + [chiffre] », renvoyant au paragraphe du vidimus (cf. note 18).

31. Le relevé repose sur la description des différents phénomènes dans M.-D. Gleßgen, « Les "lieux d'écriture"... »; voir ibid., p. 526, la liste des paramètres linguistiques traités.

32. Voir Carl-Theodor Gossen, Französische Skriptastudien, Vienne, 1967, p. 27. 
« acquitté »14 (= vid.), chateiz 14 (= vid.), prei 7 (= vid.), raportei vid. 5, vertei 16 (= vid.); Commarcei 4, 8, 10, 12 sq. (= vid.);

- l'absence de diphtongaison de /o/ accentué noté <0> issu ō de latin en syllabe ouverte : seignor 7 [= vid.], 14, signor vid. 4 sq., 18; disor 4 [= vid. + 4], ainsi que dans les formes accentuées du pronom pers. lor 5 [bis], 11, 14 (jamais lour ou leur) ; cf. aussi le pronom accentué nos 1, 15 [= vid. + 1, 3, 22] (jamais nous, 3 fois nus dans le vid.).

Les variantes vocaliques en prétonie semblent en revanche moins pertinentes :

-davant 10, 15 [= vid.], Commarcei 4, 8, 10, 12 sq. [= vid.], fasons 2 [= vid. + 2], meniere 13 [= vid., mais maniere vid. 5].

S’ajoutent les formes suivantes régionalement très marquées :

- le pronom clitique $l o 12$ (contre 4 fois $l e$ et 12 fois $l e$ art.);

- les formes verbales ai «a » (avoir, prés. 3) 8; 12 (=vid.) et at «a 》 (avoir, prés. 3) : 14 [= vid.], de même que fuit « fût » 17 (= vid.);

- le maintien de -iens dans la $4^{\mathrm{e}}$ personne verbale : les imparfaits disiens vid. 5 et estiens 6 [= vid.].

Par ailleurs, on peut noter la voyelle de transition dans ceaus «ceux 》 $(2$ [= vid. + 2]), le $e$ svarabhaktique dans le futur deverent (13 [= vid.]), la notation <ei> dans chateiz (< CAPITALES, 14 [= vid.]) ou encore la syncope dans vertei $(16$ [= vid.]).

Le vidimus suit son modèle pour la grande majorité des formes, mais il montre les phénomènes de neutralisation prouvés déjà par $\mathrm{H}$. Goebl à l'exemple des chartes copiées en Normandie ${ }^{33}$; ceux-ci concernent néanmoins exclusivement le $i$ parasite qui est omis dans cinq cas sur dixhuit :

Barvid. 9 (+5) pour Bair, chose(s) vid. 20 sq. $(+22)$ pour choises, set vid. 21 pour seit, estable vid. 20 pour estaible, letres vid. $6(+2,5,22)$ pour leitres.

Les parties nouvellement introduites par le vidimus gardent par ailleurs un marquage graphématique très reconnaissable (cf., en sus des phénomènes déjà notés, les formes avri «avril » vid. 23 et nieuf «neuf » 23).

La régionalité est nettement moins marquée dans le lexique où n'interviennent que les deux formes bien connues de la scripta documentaire lorraine junet «juin » (n.m.rég. 17 [= vid.]) et miliares « millénaire » (n.m.suj. 17 [= vid. +23$])$.

33. Hans Goebl, Die normandische Urkundensprache : ein Beitrag zur Kenntnis der nordfranzösischen Urkundensprache des Mittelalters, Vienne, 1970 (Sitzungsberichte der Österreichischen Akademie der Wissenschaften, Phil.-hist. Kl.). 
S'ajoutent le toponyme délexical (la) horne ${ }^{34}$ et, plus généralement, l'enracinement local des éléments onomastiques ${ }^{35}$.

Si nous comparons ces deux chartes aux 287 autres chartes antérieures à 1265 conservées aux archives départementales de Meurthe-et-Moselle passant donc de la microscopie à la macroscopie -, il s'avère que leur degré de régionalité dans les marques graphématiques et morphologiques est un peu supérieur à celui des grandes chancelleries laïques, celles des ducs de Lorraine et des comtes de Bar, mais bien moins développé que celui des scribes mineurs, qui sont toutefois moins présents dans notre corpus. On constate aussi que la combinaison très spécifique de leurs particularités ne se retrouve dans aucune autre charte ${ }^{36}$.

2. Les rédacteurs dans l'entourage de l'évêque de Toul. - Avant d'entreprendre l'étude graphématique systématique des lieux d'écriture, nous avions déjà opéré une comparaison détaillée entre les chartes 7 et 11 et toutes celles qui font intervenir l'évêque Roger de Toul ou un des représentants de son évêché. À cette fin, nous avons essayé de démêler les différents acteurs propres ou proches de l'évêché qui pouvaient éventuellement avoir accès à l'écrit et agir comme rédacteurs, et qui sont, pour la plupart, mentionnés dans notre charte. Ces instances, bien connues des historiens, ressortent pour la plupart du répertoire lexicologique (voir ci-dessus, III, 3) 37 . Dans une perspective centrée sur les rédacteurs potentiels, on distingue quatre entités :

(1) L'évêché à proprement parler, avec l'évêque (aveques 1), l'archidiacre (arcediacres 3 ), le chapitre (chapitre 4 etc.) des chanoines (chanenne 3, chanoinne vid. 3, 7) de l'église cathédrale et son supérieur, le doyen (doens vid. 1, 22). Les chartes ne permettent pas d'établir la moindre séparation entre ces différents acteurs, qui participaient donc à un seul scriptorium.

(2) L'officialité (officials de la cort de Toul [passim dans le corpus de $\mathrm{MM}]$ ), chargée de l'exercice judiciaire concret dans le diocèse et répondant, selon les diocèses, à l'évêque ou - ce qu'il faut supposer pour Toul - à un archidiacre. Les chartes rédigées par l'officialité se distinguent nettement, d'un point de vue paléographique, des chartes rédigées par l'évêque ou le chapitre, même si, linguistiquement, aucune différence n'est perceptible.

34. Voir FEW 16,227a, s.v. germ. *horn: Vosges du Sud : «treuil d'un puits ».

35. Les noms d'origine, comme (Symons de) Ramonchamp 3, (Bertremeus de) Apremont 3 ou (Phelipes de) Euvile 3, ont une valeur évocatrice régionale; parmi les premiers noms, notons les noms des deux serfs mentionnés, Girboles 12 et Undeneis 12, qui se détachent des noms des membres de la noblesse ou du patriciat laïque et ecclésiastique.

36. M.-D. Gleßgen, «Les "lieux d'écriture"... », p. 504-509 (« La "norme évolutive" de l'EpToul»).

37. Voir G. Bönnen, Die Bischofsstadt Toul..., p. 346-348, 374-403. 
(3) Les paroisses concernent les doyens de chrétienté (doiens de la crestientei [passim dans le corpus de MM]; lat. decani rurales) et, sous leur autorité, les différents prêtres d'une paroisse ou d'un ensemble de paroisses. Dans nos chartes, ces personnages pourtant omniprésents ne jouent qu'un rôle très restreint et la qualité de leurs productions reste faible.

(4) Les autres institutions touloises : à Toul comme ailleurs existaient de nombreuses autres églises collégiales (comme Saint-Gengoult) avec leur propre chapitre et doyen, ou des établissements réguliers (comme les Dominicains de Toul) avec leur abbé ou prieur. Le clergé toulois intervient à plusieurs reprises dans le corpus, parfois même en collaboration avec le scriptorium épiscopal qui a pu préparer des parchemins pour des abbayes (identifiables notamment par une réglure, voir ci-dessous, chartes 49,83 ). Même si les textes des différentes institutions ecclésiastiques touloises peuvent laisser entrevoir des traits propres (paléographiques et linguistiques), ils montrent une unité certaine, en opposition nette avec l'évêché.

3. L'épiscopat de Roger et la production de chartes françaises. - Pendant l'exercice spirituel de Roger de Mercy (1231-1253), onze autres chartes du corpus font intervenir l'évêque; elles sont énumérées ci-après en indiquant chaque fois le rédacteur qui a pu être déduit des divers éléments externes (mise en page, écriture) et internes (protagonistes, éléments linguistiques) ${ }^{38}$ :

- charte 9 (1239), bénéficiaire : chapitre cathédral de Toul; rédacteur (et auteur) : Gautier voué de Toul ; les formes linguistiques sont fortement régionalisées, comme il est habituel chez les scribes travaillant en dehors d'un lieu d'écriture constitué : ATA > eie [non ee], seint [non saint], varront « verront 》, estauvle « estable», lo, geu «je», mi «moi » 39 ; contrairement aux chartes 7 et 11, la charte 9 ne présente pas de $i$ parasite et note touz (vs toz) ou seignour (vs seignor); ce fort marquage et une mise en page peu soignée excluent l'évêque de Toul comme rédacteur.

- ch. 17 (1242), auteur : Roger ; rédacteur vraisemblable : comte de Bar (bénéficiaire; la charte est conservée dans la layette de «Sancy et Pierrepont», donc dans les fonds de la noblesse lorraine); Roger intervient à côté d'autres dignitaires ecclésiastiques de Toul, Metz et Lunéville ainsi que de différents seigneurs pour garantir une paix entre le comte de Bar et un de ses vassaux (le seigneur de Riste);

38. Les éditions et reproductions photographiques des chartes sont publiées sur le site des Documents linguistiques de la France, édition électronique (l'accès le plus rapide se fait par les onglets « Les corpus textuels / Table des dates »: ChMM $+\mathrm{n}^{\circ}$ de la charte). L'argumentation pour les différents documents a pris appui, dans un premier temps, sur D. Kihaï, Identification des rédacteurs... (cité note 2), p. 21, 34-36; elle repose sous sa forme actuelle sur M.-D. Gleßgen, « Les "lieux d'écriture"... », où les différents paramètres linguistiques sont présentés ; une partie des chartes en question y ont fait l'objet d'une discussion, d'autres sont présentées pour la première fois ici (ch. 29, 31, 53, 86, 110).

39. M.-D. Gleßgen, « Les "lieux d'écriture"... », p. 509. 
les éléments linguistiques conviennent à la chancellerie comtale (variété neutralisée, que [non ke], chose [non chouse], le [non lo(u)] etc.; alors que la ch. 18, liée elle aussi à cette paix, est due à un scribe du seigneur de Riste), de même que l'écriture, rapide mais professionnelle ${ }^{40}$.

- ch. 29 (1243/44), sceaux : Roger, à côté de l'évêque de Metz, de l'archevêque de Trèves et du duc de Lorraine; auteur : duc de Lorraine; rédacteur (et bénéficiaire) : abbaye de Gorze (mise en page simple mais professionnelle, écriture arrondie caractéristique des institutions monastiques, tout comme le sceau sur lacs de soie, formes linguistiques lorraines très marquées comme ATU $><\mathrm{ei}>$ [abbei « abbé »], chouse [jamais chose], 17 fois $k e$ [jamais que], lou [non le], mi [non moi], fuit $3^{\text {e }}$ parf.) ${ }^{41}$, s'expliquant par le fait que l'évêque de Toul n'intervient que de manière périphérique dans l'acte.

- ch. 31 (1243/44), auteur : Richier archidiacre de Toul; rédacteur : soit un scribe ecclésiastique de Toul soit un scribe travaillant pour le comte de Foug (bénéficiaire de la charte, conservée dans la layette de Foug); la charte est très courte et linguistiquement très neutre, ce qui rend l'identification difficile mais plaide néanmoins pour un scribe expérimenté ; l'écriture en revanche ne ressemble à aucune charte épiscopale identifiée.

- ch. 49 (1247 n.st.), sceau : Roger, rédacteur : couvent des Dominicains de Toul (bénéficiaire de cette charte de donation, conservée dans le fonds de la maison-Dieu de Toul, héritière du couvent des Dominicains); l'évêque est «très improbable comme rédacteur d'un point de vue linguistique (ATRE > eire [non ere], PAL_ATA > ieie [non iee], ceous [non ceaus, etc.], lo, amoune [non aumone]), mais aussi paléographique (document aux lignes tracées mais simple dans la calligraphie) ${ }^{42}$; c'est un des cas où il faut supposer la préparation du document par le scriptorium épiscopal et sa rédaction par un scribe du couvent des Dominicains.

- ch. 53 (1247), auteurs : Roger, Philippe comtesse de Bar et le chevalier Ferry de Brixey; rédacteur : scribe indépendant travaillant pour Geoffroy de Vaucouleurs (bénéficiaire de la présente reprise de fief; la charte est conservée dans la layette des Fiefs de Lorraine); la mise en page peu soignée (absence de marge et d'ornements, distance variable entre les lignes), et l'écriture très irrégulière sont caractéristiques des chartes rédigées par des scribes non rattachés à des lieux d'écriture constitués; s'ajoute, outre l'habituelle diphtongue /ej/ < lat. 'A[(-ATU > $e i)$, la forme dialectalement très marquée du démonstratif ceas, qui n'apparaît dans le corpus que chez ce type de scribe.

- ch. 71 (1250), sceaux : Jacques archidiacre de Toul, le duc de Lorraine et sa femme; bénéficiaire : maison du Temple de Lunéville (la charte est aussi conservée dans le fonds de la commanderie); rédacteur : duc de Lorraine ${ }^{43}$; l'archidia-

40. Ibid., p. 467.

41. La charte parallèle, ch. 30, a été rédigée en revanche par le scriptorium épiscopal de Metz : voir ibid., p. 509 et suiv.

42. Comme je l'ai indiqué ibid., p. 510.

43. Ibid., p. 501 et ill. p. 536. 
cre n'intervient qu'au titre de témoin (Jakes arcediacres de Toul qui fui presens à l'aquitance des dimes davant diz i-a mix le sien seel);

- ch. 83 (1251) et $110(1254 / 55)$; pour la ch. 83 le chanoine Hugues de Toul est le bénéficiaire, et pour la ch. 110 il est l'auteur; par la première, Morel, chancelier de Toul, lègue sa maison à Hugues, moyennant une rente importante à verser à la cathédrale lors de son anniversaire (ce qui explique pourquoi les deux chartes sont conservées dans le fonds du chapitre de Toul); par la deuxième, Hugues cède maison et rente, après la mort de Morel, à son oncle homonyme, entre-temps devenu doyen de Saint-Gengoult. La première charte est écrite avec un certain soin, néanmoins avec des éléments graphématiques qui plaident contre le centre épiscopal (tols «tous», chouse(s), citein «citoyen», cels, -v-comme marqueur d'hiatus [avost], occasionnellement chanoigne); la deuxième montre une cursive très peu soignée, en net décalage avec les chartes épiscopales peu postérieures, même simples. Puisque la première charte est placée sous le sceau du doyen de Saint-Gengoult (Jean, prédécesseur de Hugues) et puisque la mise en page avec des lignes réglées et de larges marges renvoie à l'évêché, «nous pouvons supposer que la charte ait été préparée dans le scriptorium épiscopal [...] et rédigée par un scribe de la collégiale Saint-Gengoult » ${ }^{44}$. Vu l'intérêt éminemment privé de la deuxième charte, le plus probable est que l'un des deux chanoines intéressés en fut le rédacteur.

- ch. 85 (1251), sceaux : Roger et Jean évêque de Verdun; rédacteur : évêque de Verdun; cette charte épiscopale d'apparat - il s'agit d'une copie - ressemble beaucoup aux deux chartes ici étudiées, tant dans l'écriture que dans ses motivations : il s'agit du partage des droits de marché pour Saint-Mihiel entre l'abbaye du lieu et le comte Thibaut II de Bar; les deux évêques interviennent pour garantir les prérogatives de l'institution ecclésiastique contre les velléités d'expansion du comte; les relations étroites entre l'abbaye de Saint-Mihiel et Verdun excluent néanmoins la rédaction de la charte à Toul, d'autant plus que l'évêque de Toul est introduit comme «honorable peire Rogiers », formule de révérence cohérente sous la plume d'un tiers reconnaissant, mais inhabituelle pour désigner un rédacteur; par ailleurs, les ressemblances paléographiques avec d'autres chartes d'apparat de Verdun (p. ex., ch. 14) sont flagrantes et confirmées par la physionomie des formes linguistiques, en décalage avec les documents de Toul ${ }^{45}$.

- ch. 86 (1251), sceau : Roger; rédacteur : comte de Bar (bénéficiaire); charte immédiatement reconnaissable comme un produit de la chancellerie comtale (mise en page, écriture cursive très maîtrisée et caractéristique).

La rédaction des onze chartes françaises qui font intervenir entre 1239 et 1254 l'évêque Roger ou un proche se partage équitablement entre l'univers ecclésiastique de Toul et les forces voisines : la chancellerie du comte de Bar (ch. 17, 86) et du duc de Lorraine (ch. 71), le scribe d'un seigneur local (ch. 53), le scriptorium de l'évêque de Verdun (ch. 85) et celui de l'abbaye de

44. Ibid., p. 510.

45. Ibid., p. 510 et suiv., ainsi que la forme verbale avommes [non aviens]. 
Gorze, proche de l'évêque de Metz (ch. 29). Tous les protagonistes lorrains de l'époque sont donc présents parmi les rédacteurs extérieurs. À Toul même, deux chartes ont été préparées par le scriptorium épiscopal et rédigées par des scribes de couvents moins importants (ch. 49, 83), trois sont l'œuvre d'un scribe individuel ayant reçu une formation ecclésiastique (ch. 9, 31, 110).

Les deux chartes 7 et 11 représentent donc les seules productions françaises du scriptorium de l'évêché et du chapitre. Cette réticence envers la langue vernaculaire s'affaiblira très nettement avec le successeur de Roger, l'évêque Gilles de Sorcy (1253-1269); pour les huit années allant de 12581259 à 1265, dix-huit chartes françaises du corpus de Meurthe-et-Moselle proviennent du scriptorium de l'évêché et de l'officialité épiscopale ${ }^{46}$. À l'époque où se met en place un écrit français socialement diversifié dans les documents de la Lorraine, l'évêque Roger conserve le latin comme langue presque exclusive de l'écrit, à l'instar de la chancellerie royale ${ }^{47}$. Ce constat est d'autant plus frappant que deux chartes ont été très certainement travaillées par son scriptorium avant de passer entre les mains de leurs rédacteurs; il s'agit donc d'une politique conservatrice pleinement consciente, attitude qui se retrouve en dernière instance aussi dans la mise en page et dans l'écriture livresque et archaïsante des chartes 7 et $11^{48}$.

Soulignons un dernier point : il s'agit du passage déjà cité du vidimus, « rapors est escris en letres mon signor Rogiers » (vid. 5). La forme extérieure de la charte 7 , l'écriture mais aussi la mise en page sont reconnaissables comme des productions du scriptorium épiscopal d'un évêque défini; cela confirme une nouvelle fois que les rédacteurs étaient reconnaissables par leurs contemporains et qu'ils tenaient à l'être. Si l'évêque Roger refuse, pour l'essentiel, les rédactions en français, c'est sans doute parce qu'il ne veut pas que son nom soit associé à cette forme de mise en scène du pouvoir.

Le résultat quelque peu surprenant de cette analyse souligne en même temps l'importance du choix linguistique de la charte à l'étude, choix déterminé par des objectifs politiques précis. Par ailleurs, la singularité du document explique le contraste entre une écriture certes très soignée mais en même temps archaïsante, et des marques de régionalité certes prépondérantes mais déséquilibrées (certaines formes lorraines marquées comme les $i$ parasites sont introduites dans une scripta plutôt neutralisée).

46. Ibid., p. 504.

47. Voir Paul Videsott, « À propos du plus ancien document en français de la chancellerie royale capétienne », dans le présent fascicule, p. 61-81.

48. Voir encore l'illustration dans M.-D. Gleßgen, « Les "lieux d'écriture”... », p. 530. 


\section{LECTURE HISTORIQUE.}

1. La description du terrain. - L'analyse lexicale permet d'interpréter plus en détail la description du terrain disputé entre le pouvoir épiscopal et comtal (7-10). Celle-ci détermine d'abord sa nature (7): il comporte des parties boisées (boisson), parfois difficiles d'accès (espines) ainsi que des prés (pour la production de foin) et des pâturages (prei et pastures) ; le terme de terres englobe comme archilexème les termes spécifiques qui suivent. Le terrain n'a donc pas une valeur exceptionnelle, ce qui explique pourquoi il peut avoir l'étendue que nous verrons, sans être divisé.

La localisation qui s'ensuit fournit une des rares descriptions de terrain précises de cette époque; les deux premières indications fixent le cadre général (8) :

- le ruxel devers Chairnai, qui ai nom Ruus : il doit s'agir du Rupt actuel ${ }^{49}$ qui passe par Laneuville-au-Rupt, à $2 \mathrm{~km}$ au nord-ouest de Void, pour rejoindre quelques kilomètres plus loin les méandres de la Meuse ; l'indication de la ville de Charny-sur-Meuse, au nord de Verdun, est déroutante; mais il doit s'agir d'une destination lointaine : en fait, le terrain en question connaît un partage des eaux : la partie nord et est verse ses eaux dans la Meuse (vers Verdun), la partie sud et ouest - à travers l'Ornain - dans la Marne (vers Bar-le-Duc et Paris);

- la premiere bonne k'il mirent en l'oriere do Haut Bois : la forêt du HautBois ${ }^{50}$, encore existante, culmine à $4.12 \mathrm{~m}$ et marque la ligne de partage des eaux.

Entre ces deux limites au nord (= en aval) et au sud (= en amont), la charte donne des repères plus précis $(9 s q$.) :

- tot contremont juske a-champ ke cil de Reevaus tienent do chapitre de Toul : l'indication est difficile à préciser, mais le champ doit se trouver en amont, au sud du monastère des Prémontrés de Riéval ${ }^{51}$, en aval de la «première borne »;

- contreval juke a-la-voie ke vient de Commarcei a Voi : la route de Commercy à Void ${ }^{52}$ coupe le Rupt mentionné et délimite le terrain en question vers le nord-est;

- et par desus les bonnes k'il ont assises par devant Reeval... juqe de soz La Horne, ver Voi et devers le dependant: les bornes «devant Riéval»

49. Voir pour le lexème, très fréquemment onymisé en Lorraine, FEW 10,422a s.v. RĪvUs : ru (Chrestien-Mon 1636)... ruys ([1345], Runkewitz)... Meuse rü (p. 143, 153); Gdf 7,256c-257b; 262a/b.

50. Lieu-dit, arr. Commercy, cant. Void, comm. Broussey-en-Blois.

51. Comm. Ménil-la-Horgne (Félix Liénard, Dictionnaire topographique du département de la Meuse, comprenant les noms de lieu anciens et modernes, Paris, 1872, p. 194).

52. Arr. Commercy. 
auront été posées du côté de l'accès principal de l'abbaye (côté nord); le terrain les dépasse vers l'ouest, jusqu'à la côte menant au village actuel de Ménil-la-Horgne ${ }^{53}$ (415 m, toujours sur la ligne de partage des eaux) et vers l'est jusqu'aux limites communales de Void et des hameaux qui en dépendaient.

La carte ci-après montre bien la ligne de partage des eaux qui passe par les sommets indiqués, le tracé des rivières et la position des autres toponymes. Le terrain délimité grâce aux indications de la charte devait occuper plus de dix kilomètres carrés.

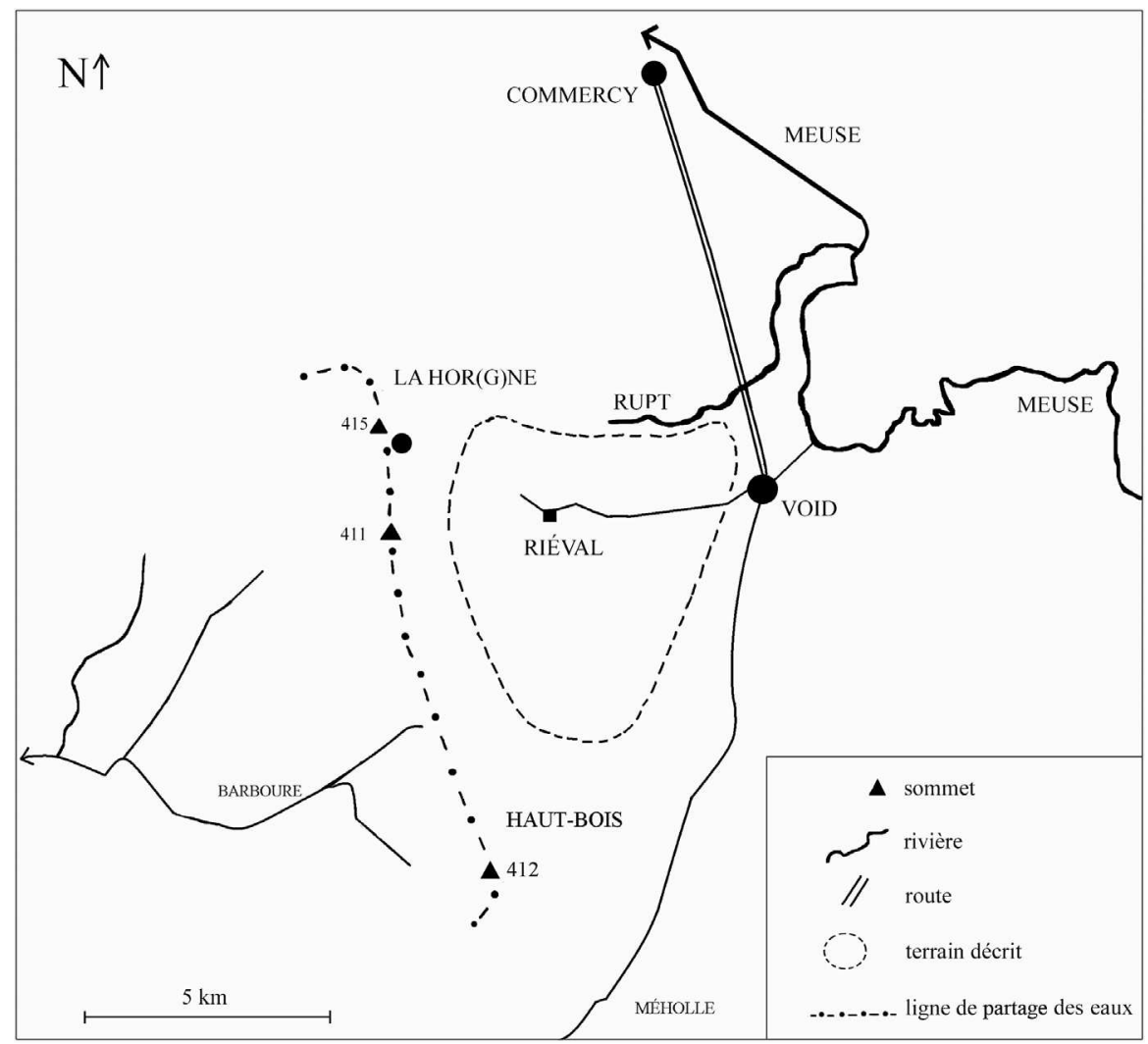

53. Arr. Commercy, cant. Void ; voir F. Liénard, Dictionnaire topographique..., p. 147, Ménil-la-Horne, attestations depuis 1586 (Menil-la-Horgne-de-Villebois) ; p. 111, (La) Horgne, attestations uniquement médiévales $(1402, \operatorname{Horn}(i) a)$; les deux noms se réfèrent sans doute au même village. 
2. Les enjeux de la charte. - L'analyse philologique permet enfin une lecture historique de la charte.

(1) Comme point de départ, le chapitre de Toul possède un terrain de plusieurs kilomètres carrés, à $25 \mathrm{~km}$ à l'ouest de la ville épiscopale, près du village de Void(-Vacon), lui-même situé à $10 \mathrm{~km}$ au sud de Commercy (div. 7-10).

(2) En 1237 ou peu avant, le comte Henri II de Bar prend en garde ce terrain (div. 5; voir ci-dessus, III, 2), opération essentiellement à son avantage puisqu'elle lui rapporte dorénavant une partie des revenus de ces terres; il investit son vassal, le seigneur Gaucher de Commercy, proche des lieux, du privilège d'en percevoir le cens (cf. vid. 12).

(3) Le seigneur de Commercy profite de ce nouveau pouvoir pour s'imposer sur le terrain (11) ainsi qu'auprès des serfs ruraux de Void, qui relèvent du chapitre, en en prenant deux à son service personnel (12).

(4) Face à ces $\operatorname{tor}(t) s(5,11)$, le chapitre devance l'opération de perception en prélevant sur les serfs les redevances que le seigneur de Commercy voudrait s'approprier (chateiz... pris a-tort en lor homes de-Void, 14).

(5) Face à cette pression, Gaucher accepte alors un arbitrage qui établit les limites de sa mainmise : il doit respecter l'intégrité du terrain (le terres... apartiennent a-chapitre de Toul, 7-10) et des hommes (il [= les arbitres] lo [= le chapitre] revestirent de deus homes, 12).

(6) Vingt-deux mois plus tard, le chapitre délivre à Gaucher un vidimus très soigné de cet arbitrage (avons donee audit Gaucher letres saelees de notre seel, vid. 22), sans doute parce que son exécution repose encore sur un équilibre fragile avec les accords pris.

L'arbitrage qui permet de reconstituer ce conflit est le fait des hommes d'Église : il fait certes intervenir aux côtés de l'archidiacre et d'un chanoine deux membres de la noblesse locale, le seigneur d'Apremont, d'une famille relativement importante, et le chevalier d'Euville, de rang mineur; mais l'évêque est investi du rôle d'arbitre suprême (estiens ellui apardesuis c'il decordoient, 6) et le scriptorium épiscopal produit les deux chartes très élaborées dont la fonction comminatoire semble évidente.

Notre charte et son vidimus témoignent d'une forte cohésion entre l'évêque et ses agents immédiats, l'archidiacre, le doyen et les chanoines, qui agissent dans la plus parfaite harmonie. Ils reflètent ainsi un moment de pouvoir bien affirmé : la construction de la nouvelle cathédrale SaintÉtienne a commencé en 1220; les forces urbaines ne prendront de la vigueur qu'au XIv ${ }^{\mathrm{e}}$ siècle et les principautés des comtes de Bar et des ducs de Lorraine n'ont pas encore atteint leur poids maximal dans la région. La situation changera considérablement vers la fin du XIII ${ }^{\mathrm{e}}$ siècle, mais au moment de la rédaction de notre charte, ses auteurs jouissent encore d'une toute-puissance temporelle. 
3. Le choix de la langue. - Au XIII ${ }^{\mathrm{e}}$ siècle, époque de transition linguistique, le choix de telle ou telle langue répond à des raisons multiples et doit être interprété au cas par cas. Ici, le français est introduit de façon délibérée à la place du latin, langue utilisée normalement dans le scriptorium épiscopal de Roger. La langue vernaculaire intervient alors comme un instrument de pouvoir : une meilleure compréhension du texte permet de mieux atteindre les destinataires, le seigneur de Commercy et le comte de Bar, que la présentation d'un document en latin, certes langue de prestige mais d'un accès plus difficile. La volonté d'être compris est motivée ici par celle d'asseoir un pouvoir temporel. Cela vaut tout autant pour la charte 85 , rédigée par le scriptorium épiscopal de Verdun, dans la même visée de contenir la force d'expansion du comte de Bar (voir ci-dessus, IV, 2). Le choix particulier de la langue vernaculaire produit donc une plus-value pragmatique dans l'antagonisme qui règne entre les pouvoirs ecclésiastique et laïque. $* *$

L'analyse de notre charte ne révolutionne en rien ni la méthodologie de la philologie ni la connaissance de l'ancienne langue. Mais elle a le mérite, en se prêtant à un regard en microscopie, de mieux asseoir l'étude des chartes et des lieux d'écriture dans cette région au XIII ${ }^{\mathrm{e}}$ siècle.

Par ailleurs, la lecture philologique des textes documentaires contient un potentiel indéniable pour la science historique : la réflexion sur les lieux d'écriture démontre la syntonie des différents acteurs dans le noyau épiscopal, qui ne saurait aller de soi; l'analyse textuelle précise les voies et les instruments de l'opposition entre les pouvoirs ecclésiastique et laïque; elle fait ressortir notamment la possible fonction comminatoire des chartes d'apparat épiscopales.

Soulignons enfin que, s'il est impossible de traiter en détail toutes les chartes lorraines ne serait-ce qu'antérieures à 1270, puisqu'elles dépassent le chiffre de mille, il est tout aussi impossible d'affronter ce patrimoine sans se livrer à la lecture détaillée d'un certain nombre d'entre elles.

Martin-D. GLessgen. 